\title{
Case Report \\ Resistant Thyrotoxicosis in a Patient with Graves Disease: A Case Report
}

\author{
Taimur Saleem, ${ }^{1}$ Aisha Sheikh, ${ }^{2}$ and Qamar Masood ${ }^{2}$ \\ ${ }^{1}$ Medical College, Aga Khan University, Stadium Road, Karachi 74800, Pakistan \\ ${ }^{2}$ Section of Diabetes, Endocrinology, and Metabolism, Department of Medicine, Aga Khan University, Stadium Road, \\ Karachi 74800, Pakistan
}

Correspondence should be addressed to Taimur Saleem, taimur@gmail.com

Received 4 March 2011; Revised 22 June 2011; Accepted 23 June 2011

Academic Editor: Fausto Bogazzi

Copyright (c) 2011 Taimur Saleem et al. This is an open access article distributed under the Creative Commons Attribution License, which permits unrestricted use, distribution, and reproduction in any medium, provided the original work is properly cited.

\begin{abstract}
Background. Conventional management of thyrotoxicosis includes antithyroid drugs, radioactive iodine, and surgery while adjunctive treatment includes beta-blockers, corticosteroids, inorganic iodide and iopanoic acid. Very rarely, patients may be resistant to these modalities and require additional management. Case Presentation. A 50-year-old lady presented with weight loss and palpitations diagnosed as atrial fibrillation. Her past history was significant for right thyroid lobectomy for thyrotoxicosis. Thyroid functions tests at this presentation showed free T4 of $6.63 \mathrm{ng} / \mathrm{dl}$ (normal range: $0.93-1.7$ ) and TSH of $<0.005 \mu \mathrm{IU} / \mathrm{mL}$ (normal range: 0.4-4.0). She was given aspirin, propranolol, heparin and carbimazole; however free T4 failed to normalize. Switching to propylthiouracil (PTU) did not prove successful. She was then given high doses of prednisolone $(1 \mathrm{mg} / \mathrm{kg} / \mathrm{day})$ and lithium (400 mg twice daily) which prepared the patient for radioactive iodine treatment by reducing free T4 levels ( $2.82 \mathrm{ng} / \mathrm{dl})$. Two doses of radioactive iodine were then administered 6 months apart. Subsequently she became hypothyroid and was started on thyroid replacement therapy. Conclusion. This case highlights management options in patients with resistant thyrotoxicosis. Radioactive iodine and surgery are definitive modes of treatment in such complex cases while steroids and lithium play an important role in preparing patients for more definitive treatment.
\end{abstract}

\section{Background}

Thyrotoxicosis affects approximately $2 \%$ of women and $0.2 \%$ of men [1]. Graves disease is the most common cause of thyrotoxicosis. It is an autoimmune disorder characterized by a constellation of clinical features including hyperthyroidism, diffuse goitre, ophthalmopathy, and dermopathy [2].

Conventional principal management of thyrotoxicosis includes antithyroid drugs, radioactive iodine, and surgery. Adjunctive treatment in the form of beta-blockers, corticosteroids, inorganic iodide, and iopanoic acid may also be used for more prompt control of symptoms [3-6]. However, a few cases may require additional treatment despite these conventional modalities to achieve euthyroid state.

We have described a case of thyrotoxicosis in a patient with Graves disease that was resistant to antithyroid drugs. High-dose steroids and lithium were additionally administered to improve her thyrotoxicosis so that radioactive iodine could be administered later.

\section{Case Presentation}

A 50-year-old lady from Afghanistan presented to our institution with complaints of undocumented weight loss, palpitations, and anxiety for the past 4 weeks. Her past history was significant for right thyroid lobectomy for thyrotoxicosis more than four years ago. She did not have any history of a recent iodine load. Clinically, she had an irregularly irregular pulse of 110-120 beats per minute. Physical examination showed signs of thyrotoxicosis, a diffusely enlarged left thyroid lobe and thyroid bruit. Her electrocardiograph (ECG) showed atrial fibrillation with rapid ventricular rate (RVR). Thyroid functions tests were ordered which showed severe thyrotoxicosis with free T4 of $6.63 \mathrm{ng} / \mathrm{dl}$ (normal range: $0.93-$ 1.7) and TSH of $<0.005 \mu \mathrm{IU} / \mathrm{mL}$ (normal range: $0.4-4.0$ ). A diagnosis of Graves disease was made on the basis of high titres of thyroid antibodies (antithyroid peroxidase antibody $>1000 \mathrm{IU} / \mathrm{mL}$ (normal range: $0-12$ )). In addition, the presence of a thyroid bruit is also considered pathognomonic for 
Graves disease. The patient did not have any signs of Graves ophthalmopathy or dermopathy.

Echocardiography showed moderately dilated right atrium with a severely dilated left atrium and moderate to severe mitral regurgitation and no evidence of a thrombus. Her left ventricular systolic function was moderately reduced with estimated ejection fraction of $35-40 \%$. Ultrasound of the neck showed heterogeneously enlarged left lobe of thyroid gland which measured $4 \times 3.5 \mathrm{~cm}$; no discrete nodules were identified.

It was planned that the patient be made euthyroid with free T4 values as near normal as possible, so that radioactive iodine (RAI) could be safely administered without the risk of precipitating thyrotoxic crises. The patient was started on aspirin, propranolol, heparin, and carbimazole. Her initial dose of carbimazole was $30 \mathrm{mg} /$ day but was increased up to $90 \mathrm{mg} /$ day due to no improvement in symptoms. Her free T4 failed to normalize despite maximum doses of carbimazole. Switching to propylthiouracil (PTU) and administering maximum doses had minimal benefit with free T4 values still bordering on $4 \mathrm{ng} / \mathrm{dl}$. The patient was an illiterate lady who was accompanied by her son at every visit. The issue of compliance was thoroughly ascertained through direct questioning and visual inspection of pill bottles at the initial visit and then at every subsequent visit thereafter. The patient appeared to be compliant in taking her medications.

She was then given high doses of prednisolone $(1 \mathrm{mg} / \mathrm{kg} /$ day) along with lithium (400 mg twice daily) to make her euthyroid so that RAI could be safely given in the future. The patient showed a good response to these treatment modalities, and her free T4 further reduced to $2.82 \mathrm{ng} / \mathrm{dl}$. However, despite intensive medical management, her thyrotoxic symptoms showed little improvement overall.

PTU was stopped 5 days before giving the RAI to enhance its uptake by the thyroid. After a period of 12 weeks from the initial presentation to our institution, the patient was administered $15 \mathrm{mCi}$ of RAI. Unfortunately, measurement of radioactive iodine uptake prior to the administration of RAI is not performed at our institution.

She was then given potassium iodide for the next two weeks. Ross et al. [7] have shown that adjunctive potassium iodide given after RAI has the potential to treat thyrotoxicosis more rapidly than RAI alone without any adverse effect on outcomes at 1 year. Although not standard practice yet, this particular method of treatment [7] has been utilized by at least 19 authors in the management of patients with thyrotoxicosis. As our patient was resistant to antithyroid drugs, we explored all available options including administration of potassium iodide for a short duration of time.

The patient remained stable without the occurrence of thyrotoxic crises. After 3 days, PTU was restarted at a dose of $1200 \mathrm{mg}$ /day. Her free T4 measured at three months after the RAI was $2.93 \mathrm{ng} / \mathrm{dl}$. After 6 months of initial administration of RAI, the patient was still thyrotoxic. A second dose of $20 \mathrm{mCi}$ of RAI was then administered.

Three weeks after receiving the second RAI dose, her free $\mathrm{T} 4$ decreased to $1.81 \mathrm{ng} / \mathrm{dl}$. Another repeat measurement in three weeks showed a free T4 value of $0.87 \mathrm{ng} / \mathrm{dl}$. The patient was started on thyroid replacement therapy (at $50 \mathrm{mcg} /$ day) soon thereafter as she had become hypothyroid. Her clinical condition has remained stable at 6 months followup.

\section{Discussion}

3.1. Causes of Thyrotoxicosis. Graves disease is the most common cause of thyrotoxicosis. Thyrotoxicosis can also be associated with other entities such as toxic nodular goiter, human chorionic gonadotropin, pituitary resistance to thyroid hormone regulation, $\mathrm{TSH}$-secreting pituitary adenoma, subacute thyroiditis, ectopic hormone production, or exogenous ingestion of thyroid hormone. In addition, it can also be iodine induced [5] or may occur with postpartum thyroiditis.

\subsection{Medical Management of Thyrotoxicosis}

3.2.1. Thionamides. Methimazole/carbimazole are the mainstay of treatment in the majority of patients with Gravesassociated thyrotoxicosis. PTU, on the other hand, is considered a second-line agent (except in the first trimester of pregnancy) due to reports of associated severe hepatotoxicity. These drugs decrease thyroid hormone synthesis, and control of hyperthyroidism is usually achieved within several weeks. Large doses of PTU have been reported to decrease the peripheral conversion of $\mathrm{T} 4$ to $\mathrm{T} 3$ [3]. Thionamides also have an important role in the long-term management of patients with Graves disease. A treatment regime of 12-18 months has been reported to induce remission in $40-60 \%$ of patients [8]. Although initially championed with a vogue of enthusiasm, it has now been reported that block-replace regimen (combined thionamide and thyroxine therapy) does not increase the chances of long-term remission in patients [6]. Common side effects of thionamides include fever, urticaria, arthralgias, and mild elevations in transaminase levels while more severe side effects such as agranulocytosis, acute inflammatory hepatitis, and vasculitis are rare [3].

3.2.2. Adjunctive Drugs. In certain instances, adjunctive drugs may also be required to alleviate severe symptoms or achieve euthyroidism more promptly. Such aggressive management is usually needed in elderly patients as well as patients with thyrotoxic heart disease. Beta-blockers, highdose glucocorticoids, and iopanoic acid (an oral cholecystographic agent) all inhibit the peripheral conversion of T4 to T3 while inorganic iodide and iopanoic acid decrease synthesis and release of thyroid hormone [3]. Sodium ipodate has been used for the long-term control of Graves thyrotoxicosis and is believed to cause a more rapid fall in thyroid hormone level and provide an easier control of symptoms. However, it may itself rarely cause severe resistant hyperthyroidism when used in the treatment of Graves disease [9].

3.3. Radioactive Iodine Ablation. ${ }^{131} \mathrm{I}$ is an effective definitive treatment option for Graves thyrotoxicosis; a single dose is successful in achievement of euthyroid status in the majority of patients within 8 weeks [10]. However, ${ }^{131} \mathrm{I}$ causes permanent hypothyroidism in almost all patients in the long run $[3,6]$. Other adverse aspects associated with the use of 
${ }^{131}$ I also merit consideration; these include initial worsening of thyrotoxicosis and development or worsening of Graves ophthalmopathy [3]. In our patient, the first dose of RAI was not successful in controlling the hyperthyroid symptoms even after 6 months. Therefore, a second dose of RAI was administered. An expected side effect of this therapy occurred in our patient in the form of hypothyroidism. The patient was then put on thyroid replacement therapy.

3.4. Surgical Management of Thyrotoxicosis. Thyroidectomy is the definitive surgical management for Graves thyrotoxicosis and usually requires a short course of pretreatment with thionamides or inorganic iodine to achieve euthyroid state; this reduces operative complications and thyroid vascularity. Although subtotal thyroidectomy was once practiced, most surgeons now recommend complete thyroidectomy to minimize chances of relapse $[3,6]$. The present case also demonstrates why subtotal thyroidectomy in this setting has fallen out of favor. While the procedure is not associated with worsening of Graves ophthalmopathy, it can be complicated by bleeding, infection, recurrent laryngeal nerve injury, hypocalcemia, and permanent hypothyroidism [3].

The modus operandi selected for each patient should be individualized on a case-per-case basis. A consideration should be given to the merits and demerits of the chosen modality. Local and regional trends may also influence this decision. For example, radioiodine is the preferred primary modality in the USA while antithyroid drug therapy is preferred in Europe and Australia [6].

3.5. Resistant Thyrotoxicosis. Majority of the patients respond to the conventional treatments outlined above. However, literature review showed sporadic reports of patients with resistant thyrotoxicosis.

3.5.1. Etiologies of Resistant Thyrotoxicosis. Etiologies of resistant thyrotoxicosis in literature include type I amiodaroneinduced thyrotoxicosis (AIT) [11] and Graves disease [1216]. Refractory cases have mostly shown resistance to highdose thionamides and beta-blockers; rarely resistance to iodine has also been reported [11-13, 15-18]. In our patient, resistance to two different thionamides was seen. Also, betablockers did not improve the thyrotoxic symptoms.

3.5.2. Mechanisms of Resistance. Possible mechanisms mediating resistance in cases refractory to conventional treatment may range from drug malabsorption, rapid drug metabolism, antidrug antibodies, impairment of intrathyroidal drug accumulation or action, and predominant elevation of $\mathrm{T} 3$ rather than T4 levels [15].

3.5.3. Workup of Patients with Resistant Thyrotoxicosis. Evaluation of resistant cases should always start with the evaluation of patient compliance [17]. We were attentive to this aspect in the management of our patient and found her to be compliant in taking her medications. This compliance was ascertained through direct questioning at the initial visit and then at every subsequent visit thereafter. Malabsorption should also be ruled out in these patients through careful history taking and physical examination. In our patient, there was nothing remarkable in the history or physical examination to suggest malabsorption as a potential cause for drug resistance. The measurement of drug levels or anti-drug antibodies may then be undertaken. However, these tests are usually not available for routine clinical use; we also did not perform them at our institution due to nonavailability. Alternatively, resistance to drugs can be tested by performing a perchlorate discharge test four hours after drug intake under medical supervision. However, it should be noted that pharmaceutical-grade perchlorate may not be universally available. For example, it is no longer manufactured in the USA. Although not performed in our patient, iodine measurement of urinary iodine excretion may be additionally undertaken to evaluate iodine contamination [19].

3.5.4. Management of Resistant Thyrotoxicosis. Different approaches have been used for the management of resistant thyrotoxicosis. Such patients can either be offered surgery or radioactive iodine ablation as the definitive treatment. However, achievement of a euthyroid state before opting for any of these interventions is recommended in order to minimize potential complications such as precipitation of thyroid crisis $[13,14,17]$. Some authors have advocated the use of iopanoic acid prior to definitive thyroidectomy in patients with drugresistant Type I AIT. [11] as well as in patients with Graves disease [12]. However, iopanoic acid, like perchlorate, is no longer universally available and is no longer manufactured, for example, in the USA. Although corticosteroids have traditionally been used in the management of thyroid crisis [20], some authors have also reported their use in the management of resistant thyrotoxicosis. Dexamethasone, in conjunction with iopanoic acid, has been used for the rapid preoperative preparation of uncontrolled, resistant thyrotoxicosis [12]. Similarly, prednisolone $(20 \mathrm{mg} /$ day $)$, added to antithyroid drugs, has been successfully used to achieve euthyroid state in patients with resistant thyrotoxicosis before administration of radioiodine [17]. Cholestyramine, a bile acid sequestrant, has been shown to cause a dramatic decline in serum thyroid hormone levels in Graves associated thyrotoxicosis resistant to conventional treatment [13].

Lithium, an agent used to treat bipolar affective disorder, has shown favorable response in resistant cases of thyrotoxicosis. Lithium probably increases retention of radioactive iodine in the thyroid in treatment-resistant Graves thyrotoxicosis and has accordingly been used as an adjunct to radioactive iodine in such cases [16, 21-24]. Such use is especially beneficial in patients with underlying cardiovascular disorders in whom even minor elevations of thyroid hormone levels can prove seriously deleterious [24]. We successfully used a combination of lithium and steroids to prepare the patient for the RAI procedure.

\section{Conclusion}

This case highlights an important yet uncommon clinical entity of resistant thyrotoxicosis. Our patient was resistant to the conventional management including beta-blockers and 
thyrostatics. We have also discussed the possible management options in such patients. Radioactive iodine or surgery are the definitive modes of treatment in such complex cases while steroids and lithium may play an important part in preparing the patients for more definitive forms of treatment.

\section{Conflict of Interests}

The authors declare that they have no Conflict of interests.

\section{Author's Contribution}

T. Saleem and Q. Massod collected the data, helped in its interpretation, and drafted the paper. A. Sheikh conceived the study, helped in data collection and interpretation, drafted the paper, and provided overall supervision in the project. All authors read and approved the final paper.

\section{Consent}

Written informed consent was obtained from the patient for the publication of this case report and accompanying images. A copy of the consent is available for review by the Editor-inChief of this journal.

\section{References}

[1] J. A. Franklyn, "The management of hyperthyroidism," The New England Journal of Medicine, vol. 330, pp. 1731-1738, 2002.

[2] J. Ginsberg, "Diagnosis and management of Graves' disease," Canadian Medical Association Journal, vol. 168, no. 5, pp. 575$585,2003$.

[3] E. N. Pearce, "Diagnosis and management of thyrotoxicosis," British Medical Journal, vol. 332, no. 7554, pp. 1369-1373, 2006.

[4] J. R. Reid and S. F. Wheeler, "Hyperthyroidism: diagnosis and treatment," American Family Physician, vol. 72, no. 4, pp. 623636, 2005.

[5] J. V. Hennessey, "Diagnosis and management of thyrotoxicosis," American Family Physician, vol. 54, no. 4, pp. 1315-1324, 1996.

[6] D. J. Topliss and C. J. Eastman, "Diagnosis and management of hyperthyroidism and hypothyroidism," Medical Journal of Australia, vol. 180, no. 4, pp. 186-193, 2004.

[7] D. S. Ross, G. H. Daniels, and P. De Stefano, "Use of adjunctive potassium iodide after radioactive Iodine (131I) treatment of Graves' hyperthyroidism," Journal of Clinical Endocrinology and Metabolism, vol. 57, no. 2, pp. 250-253, 1983.

[8] P. Abraham, A. Avenell, W. A. Watson, C. M. Park, and J. S. Bevan, "Antithyroid drug regimen for treating Graves' hyperthyroidism," Cochrane Database of Systematic Reviews, no. 2, Article ID CD003420, 2004.

[9] G. Caldwell, M. Errington, and A. D. Toft, "Resistant hyperthyroidism induced by sodium ipodate used as treatment for Graves' disease," Acta Endocrinologica, vol. 120, no. 2, pp. 215216, 1989.

[10] L. E. Holm, G. Lundell, I. Dahlqvist, and A. Israelsson, "Cure rate after 131I therapy for hyperthyroidism," Acta Radiologica. Oncology Radiation Therapy Physics and Biology, vol. 20, no. 3, pp. 161-166, 1981.
[11] F. Bogazzi, F. Aghini-Lombardi, C. Cosci et al., "Iopanoic acid rapidly controls Type I amiodarone-induced thyrotoxicosis prior to thyroidectomy," Journal of Endocrinological Investigation, vol. 25, no. 2, pp. 176-180, 2002.

[12] C. K. Pandey, M. Raza, S. Dhiraaj, A. Agarwal, and P. K. Singh, "Rapid preparation of severe uncontrolled thyrotoxicosis due to Grave's disease with Iopanoic acid-a case report," Canadian Journal of Anesthesia, vol. 51, no. 1, pp. 38-40, 2004.

[13] A. Sebastián-Ochoa, M. Quesada-Charneco, D. FernándezGarcía, R. Reyes-García, P. Rozas-Moreno, and F. EscobarJiménez, "Dramatic response to cholestyramine in a patient with Graves' disease resistant to conventional therapy," Thyroid, vol. 18, no. 10, pp. 1115-1117, 2008.

[14] B. Winsa, J. Rastad, E. Larsson et al., "Total thyroidectomy in therapy-resistant Graves' disease," Surgery, vol. 116, no. 6, pp. 1068-1075, 1994.

[15] H. Li, J. Okuda, T. Akamizu, and T. Mori, "A hyperthyroid patient with Graves' disease who was strongly resistant to methimazole: investigation on possible mechanisms of the resistance," Endocrine Journal, vol. 42, no. 5, pp. 697-704, 1995.

[16] K. Hoogenberg, J. A. M. Beentjes, and D. A. Piers, "Lithium as an adjunct to radioactive iodine in treatment-resistant Graves thyrotoxicosis," Annals of Internal Medicine, vol. 129, no. 8, p. 670, 1998.

[17] E. B. Jude, J. Dale, S. Kumar, and P. M. Dodson, "Treatment of thyrotoxicosis resistant to carbimazole with corticosteroids," Postgraduate Medical Journal, vol. 72, no. 850, pp. 489-491, 1996.

[18] R. Hall and J. H. Lazarus, "Changing iodine intake and the effect on thyroid disease," British Medical Journal, vol. 294, no. 6574, pp. 721-722, 1987.

[19] B. Corvilain and P. Schinohoritis, "Carbimazole-Resistant thyrotoxicosis," Postgraduate Medical Journal, vol. 73, no. 864, p. 686, 1997.

[20] J. D. Wilson and D. W. Foster, Textbook of Endocrinology, W. B. Saunders, 8th edition, 1992.

[21] J. G. Turner, B. E. W. Brownlie, and T. G. H. Rogers, "Lithium as an adjunct to radioiodine therapy for thyrotoxicosis," Lancet, vol. 1, no. 7960, pp. 614-615, 1976.

[22] F. Akin, G. F. Yaylali, and M. Bastemir, "The use of lithium carbonate in the preparation for definitive therapy in hyperthyroid patients," Medical Principles and Practice, vol. 17, no. 2, pp. 167-170, 2008.

[23] Y. W. Ng, S. C. Tiu, K. L. Choi et al., "Use of lithium in the treatment of thyrotoxicosis," Hong Kong Medical Journal, vol. 12, no. 4, pp. 254-259, 2006.

[24] F. Bogazzi, L. Bartalena, A. Campomori et al., "Treatment with lithium prevents serum thyroid hormone increase after thionamide withdrawal and radioiodine therapy in patients with graves' disease," Journal of Clinical Endocrinology and Metabolism, vol. 87, no. 10, pp. 4490-4495, 2002. 


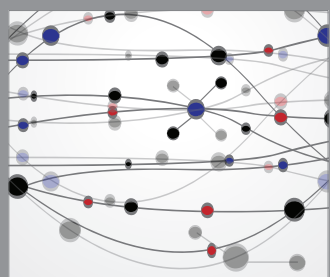

The Scientific World Journal
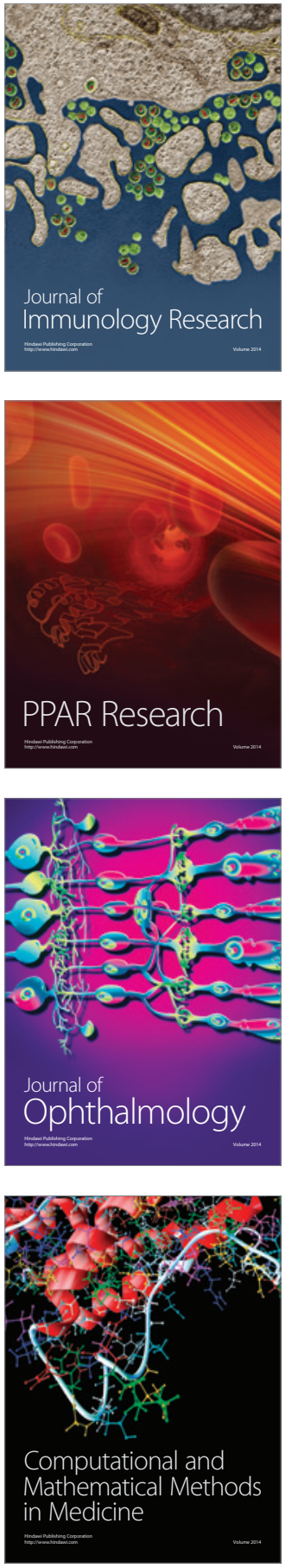

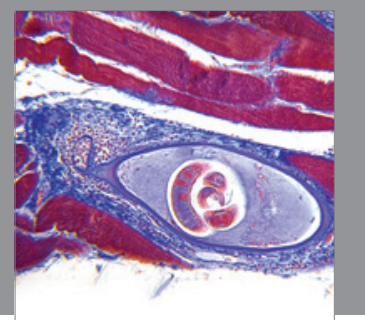

Gastroenterology

Research and Practice
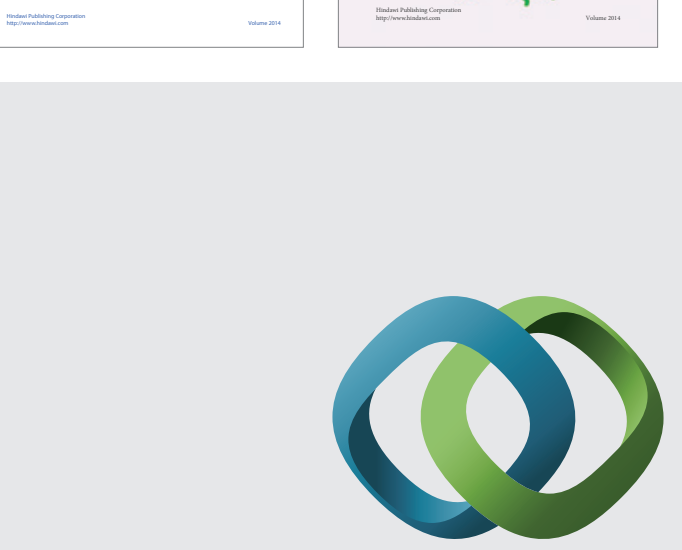

\section{Hindawi}

Submit your manuscripts at

http://www.hindawi.com
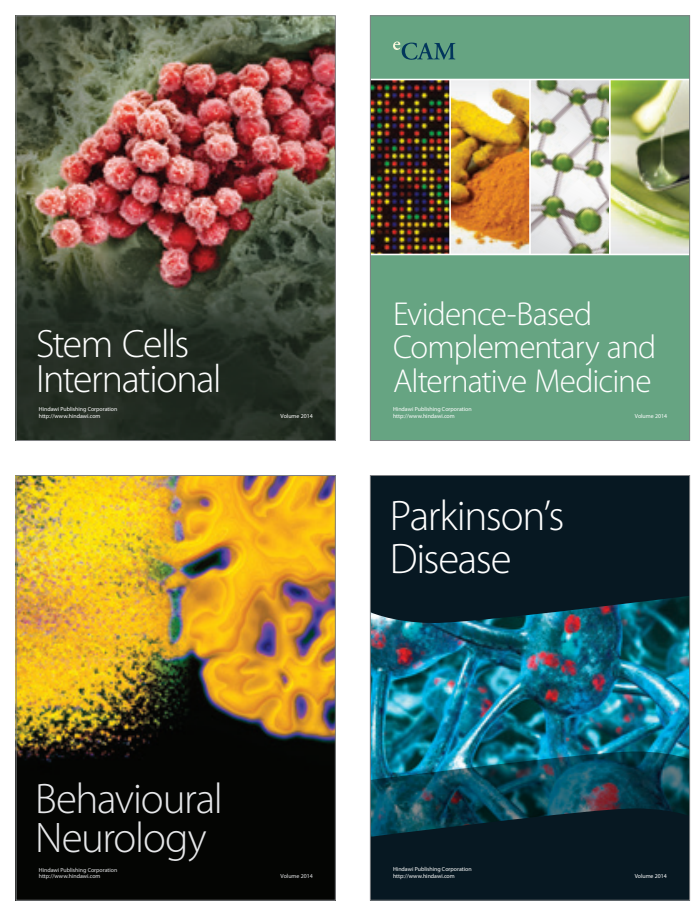

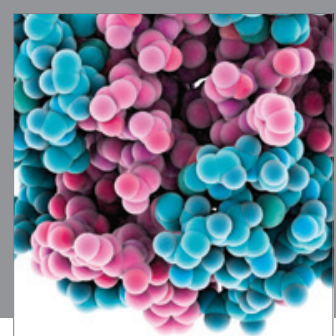

Journal of
Diabetes Research

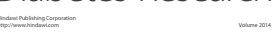

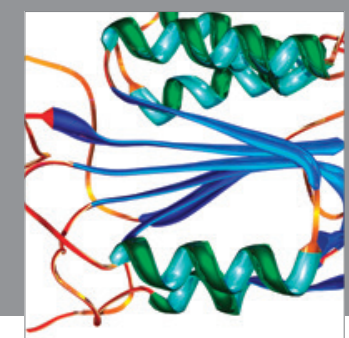

Disease Markers
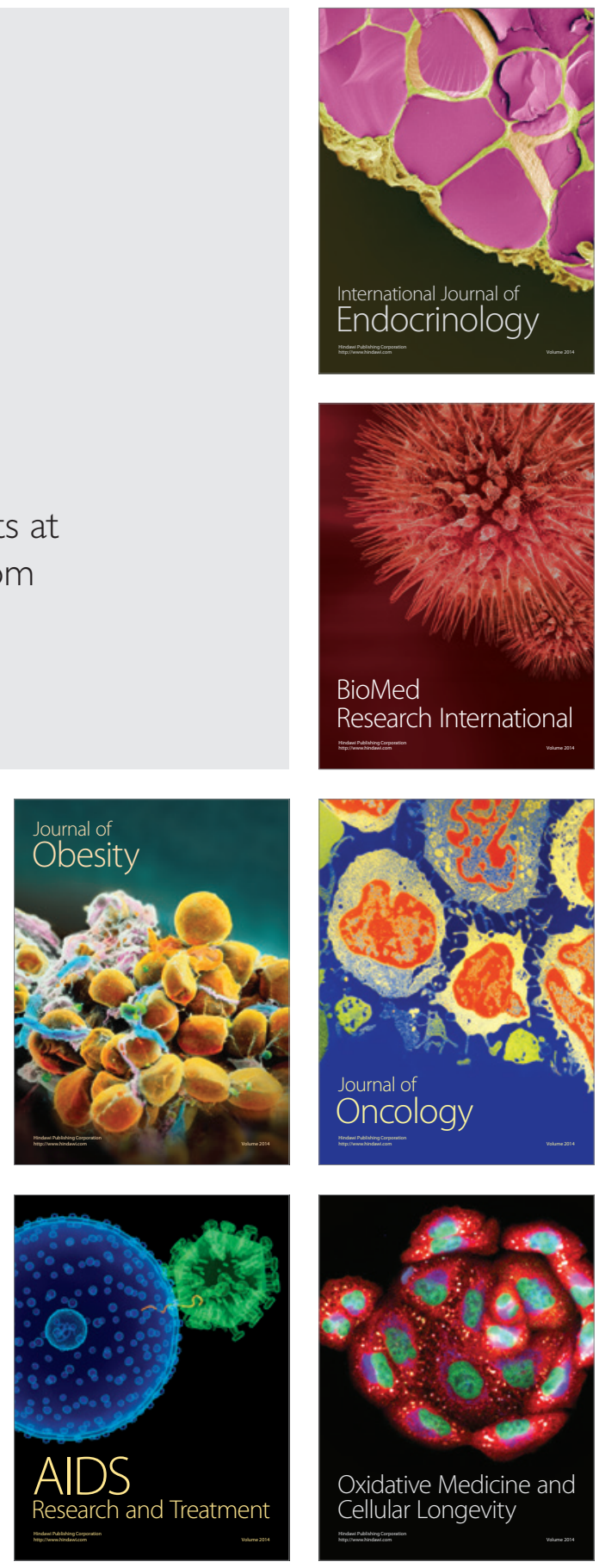